\title{
Chimie et changement climatique
}




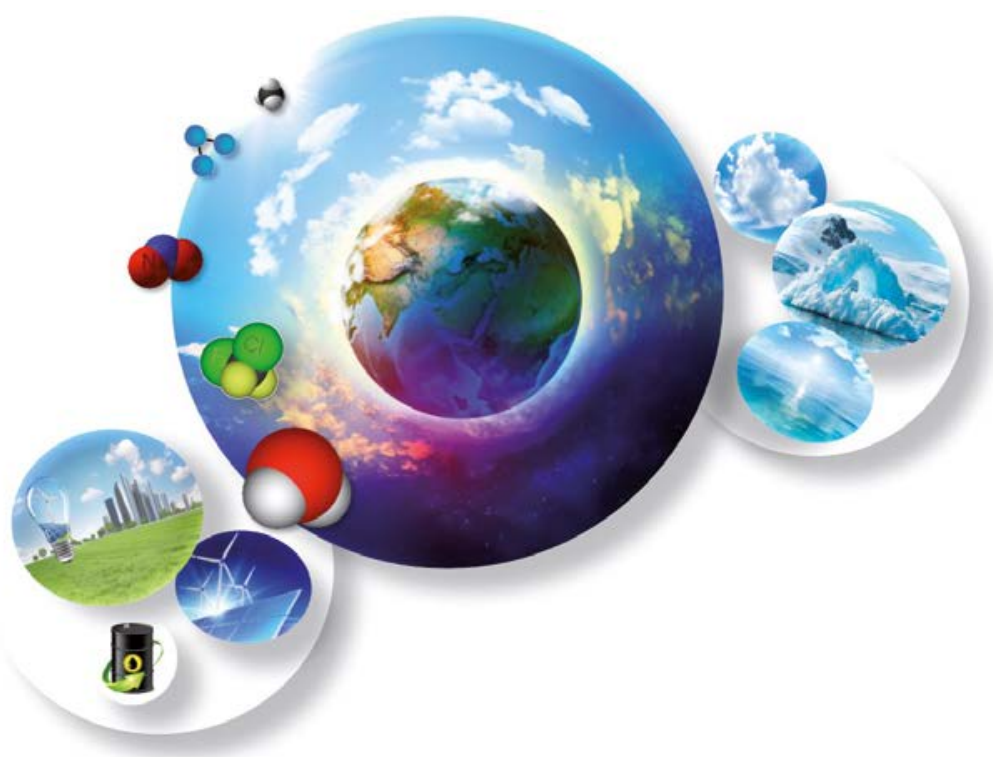

Cet ouvrage est issu du colloque « Chimie et changement climatique », qui s'est déroulé le 18 novembre 2015 à la Maison de la Chimie. 
« COLLECTION CHIMIE ET ... »

Collection dirigée par Bernard Bigot

Président de la Fondation internationale de La Maison de la Chimie
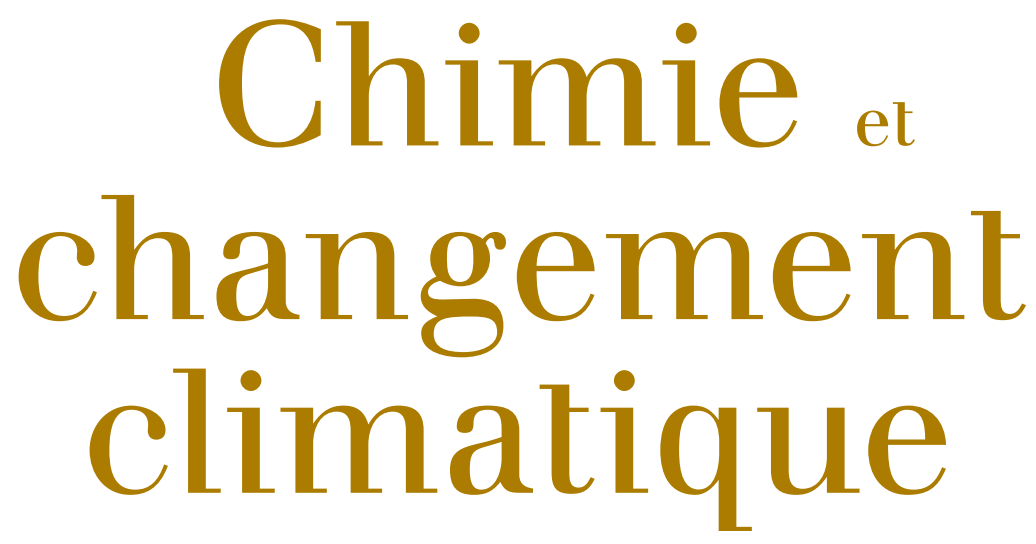

Jean-Claude Bernier, Guy Brasseur, Yves Bréchet, Sébastien Candel, Anny Cazenave, Vincent Courtillot, Marc Fontecave, Emmanuel Garnier, Philippe Gœbel, Jack Legrand, Michel Legrand, Hervé Le Treut, Pascal Mauberger

Coordonné par Minh-Thu Dinh-Audouin, Danièle Olivier et Paul Rigny 
Conception de la maquette intérieure et de la couverture :

Pascal Ferrari et Minh-Thu Dinh-Audouin

Images de la couverture : glace, ampoule, globe: doethion, Coloures-pic, Sergey Nivens - Fotolia.com ; microalgue : Wikipédia, Creative Commons CC-BY-2.5, Image courtesy of Alessandra de Martino and Chris Bowler; $4^{\mathrm{e}}$ de couverture : ampoule et ville : Coloures-pic - Fotolia.com; SG COP21; globe : Simon Chabrillat, BISA ; éoliennes : David McKay (2012).

Iconographie: Minh-Thu Dinh-Audouin

Mise en pages et couverture : Patrick Leleux PAO (Caen)

Imprimé en France

ISBN : 978-2-7598-2035-1

Tous droits de traduction, d'adaptation et de reproduction par tous procédés, réservés pour tous pays. La loi du 11 mars 1957 n'autorisant, aux termes des alinéas 2 et 3 de l'article 41, d'une part, que les «copies ou reproductions strictement réservées à l'usage privé du copiste et non destinées à une utilisation collective», et d'autre part, que les analyses et les courtes citations dans un but d'exemple et d'illustration, «toute représentation intégrale, ou partielle, faite sans le consentement de l'auteur ou de ses ayants droit ou ayants cause est illicite » (alinéa $1^{\text {er }}$ de l'article 40). Cette représentation ou reproduction, par quelque procédé que ce soit, constituerait donc une contrefaçon sanctionnée par les articles 425 et suivants du code pénal.

(C) EDP Sciences 2016 


\section{Ont contribué à la rédaction de cet ouvrage :}

\author{
Jean-Claude Bernier \\ Professeur Émérite de \\ l'Université de Strasbourg \\ Ancien directeur du \\ département Chimie du CNRS \\ Guy Brasseur \\ Ancien Directeur \\ Institut Max Planck de \\ Météorologie (Hambourg, \\ Allemagne) \\ National Center for \\ Atmospheric Research \\ (Boulder, CO, États-Unis) \\ Yves Bréchet \\ Professeur \\ Institut Polytechnique de \\ Grenoble \\ Haut-Commissaire à l'Énergie \\ Atomique \\ Membre de l'Académie \\ des Sciences
}

\section{Sébastien Candel}

Professeur des Universités

Émérite à CentraleSupélec

Laboratoire EM2C, CNRS

Université Paris-Saclay

Vice-président de l'Académie

des sciences

\section{Anny Cazenave}

Directeur pour les sciences de la Terre à l'International Space Science Institute à Berne

Chercheur Émérite au

Laboratoire d'Études en

Géophysique et Océanographie

Spatiale

Membre de l'Académie

des sciences

\author{
Vincent Courtillot \\ Professeur Émérite \\ de Géophysique \\ Université Paris-Diderot \\ Ancien Directeur de l'Institut \\ de Physique du Globe \\ Membre de l'Académie \\ des Sciences \\ Marc Fontecave \\ Professeur au Collège de \\ France \\ "Chaire de Chimie des \\ Processus Biologiques » \\ Président de la Fondation du \\ Collège de France \\ Membre de l'Académie des \\ Sciences
}

Emmanuel Garnier

Membre senior Institut

Universitaire de France

Directeur de Recherche CNRS

Historien du climat et des

risques

UMR LIENSs-Université de La

Rochelle

Philippe Gœbel

Président de l'Union des

Industries Chimiques (UIC)

Jack Legrand

Professeur à l'Université de

Nantes

Directeur du GEPEA,

Laboratoire de Génie des

Procédés, Environnement, Agroalimentaire - UMR CNRS 6144/Université de Nantes/ École des Mines de Nantes/ ONIRIS

\section{Michel Legrand \\ Directeur de recherche au \\ CNRS \\ Chimiste de l'atmosphère et des archives glaciaires Laboratoire de Glaciologie et Géophysique de \\ l'Environnement UMR 5183, Grenoble}

\section{Hervé Le Treut}

Professeur à l'Université Pierre et Marie Curie et à l'École

Polytechnique

Directeur de l'Institut-Pierre-

Simon-Laplace

Membre de l'Académie des

Sciences

Pascal Mauberger

Président Directeur Général de McPhy Energy

Président de l'Association

Française pour l'Hydrogène et les Piles à Combustible (AFHYPAC)
Équipe éditoriale :

Minh-Thu Dinh-Audouin, Danièle Olivier et Paul Rigny 
